\title{
ENUMERATION THEOREMS IN INFINITE ABELIAN GROUPS ${ }^{1}$
}

\author{
D. L. BOYER ${ }^{2}$
}

1. Introduction. W. R. Scott [2, Theorem 9] has proved that an Abelian group of order $A>\boldsymbol{\aleph}_{0}$ has $2^{A}$ subgroups of order $A$, and the intersection of all the subgroups of order $A$ is the identity. He has also proved [2, Theorem 10] that the intersection of all the infinite subgroups of a countable Abelian group $G$ is the identity unless $G=Z\left(p^{\infty}\right) \oplus F$, where $F$ is finite. In the present paper the remaining parts of Scott's Theorem 9 will be extended to countable Abelian groups ( $\$ 2$ ) by characterizing those countable Abelian groups with $\boldsymbol{\aleph}_{0}$ infinite subgroups and showing that all others have $\boldsymbol{N}$ infinite subgroups. It is also pointed out $(\$ 2)$ that the above-mentioned theorem is valid for modules over a principal ideal ring with a restriction on the order of the ring. Finally $(\$ 3)$ it is shown that the order of the automorphism group of a countable torsion Abelian group is $\mathbf{N}$. The reader is referred to [1] for the well-known theorems and definitions used. In the statements of the theorems, H. stands for hypothesis and C. stands for conclusion.

\section{Subgroups of countable Abelian groups and submodules of modules.}

Lemma 1. H. $G=Z\left(p^{\infty}\right) \oplus Z\left(p^{\infty}\right)$.

C. $G$ has $\aleph$ subgroups.

Proof. Each of the sequences of elements

$$
\begin{gathered}
(1 / p, i / p),\left(1 / p^{2},(i+j p) / p^{2}\right),\left(1 / p^{3},\left(i+j p+k p^{2}\right) / p^{3}\right), \cdots, \\
i=0,1, \cdots, p-1 ; j=0,1, \cdots, p-1 ; k=0,1, \cdots, p-1 ; \cdots
\end{gathered}
$$

generates a distinct subgroup and there are $\boldsymbol{\aleph}$ such sequences.

The following lemma concerns groups which are not necessarily Abelian.

Lемма 2. H. $H$ is a finite normal subgroup of a group $G$. The number

\footnotetext{
${ }^{1}$ This paper is taken from the author's thesis which has been submitted to the faculty of the Graduate School of the University of Kansas in partial fulfillment of the requirements for the Ph.D. degree. The author is indebted to Professor W. R. Scott, his thesis advisor. In particular, Lemma 2 is due to him.

2 This work was supported by the National Science Foundation through Research Grant NSF-G1126.
} 955. 
of subgroups of $G / H$ is $A \geqq \aleph_{0}$.

C. The number of subgroups of $G$ is $A$.

Proof. Since each subgroup of $G / H$ is associated with a subgroup of $G, G$ has at least $A$ subgroups. Assume that $G$ has more than $A$ subgroups and let $\left\{K_{\alpha}\right\}$ be the subgroups of $G$. Notice first that there is some $G_{1} \subset G$ such that $H \cup K_{\alpha}=G_{1}$ ( $\cup$ is the group-theoretic union) for more than $A$ of the $K_{\alpha}$; for otherwise, since $G / H$ has $A$ subgroups, there would be only $A$ of the $K_{\alpha}$. Also, $H$ is normal in $G_{1}$ and the number of subgroups of $G_{1} / H$ is less than or equal to the number of subgroups of $G / H$ which is $A$. Since $H$ is finite and $H \cup K_{\alpha}=G_{1}$, the index of $K_{\alpha}$ in $G_{1}$ is finite. Hence for each $K_{\alpha}$, there exists an $N_{\alpha} \subset K_{\alpha}$ such that $N_{\alpha}$ is normal in $G_{1}$ and the index of $N_{\alpha}$ in $G_{1}$ is finite. Thus there are only a finite number of the $K_{\alpha}$ such that $N_{\alpha} \subset K_{\alpha} \subset G_{1}$, i.e. only a finite number of the $K_{\alpha}$ can correspond to a given $N_{\alpha}$. Hence there are more than $A$ of the $N_{\alpha}$.

Now there is a subgroup $G_{2} \subset G_{1}$ and a subgroup $H_{1} \subset H$ such that $H \cup N_{\alpha}=G_{2}$ and $H \cap N_{\alpha}=H_{1}$ for more than $A$ of the $N_{\alpha}$, for otherwise there would be at most $A$ of the $N_{\alpha}$. Also notice that $H_{1}$ is normal in $G_{2}$. The situation is now as follows:

$H / H_{1}$ is finite and normal in $G_{2} / H_{1}$.

$\left(G_{2} / H_{1}\right) /\left(H / H_{1}\right) \cong G_{2} / H$ and $G_{2} / H$ has at most $A$ subgroups. $\left(N_{\alpha} / H_{1}\right) \cup\left(H / H_{1}\right)=G_{2} / H_{1}$ and $\left(N_{\alpha} / H_{1}\right) \cap\left(H / H_{1}\right)=H_{1} / H_{1}$ for more than $A$ of the $N_{\alpha}$; thus the index of $N_{\alpha} / H_{1}$ in $G_{2} / H_{1}$ is finite.

For a fixed $\alpha_{0}$, let $P_{\beta} / H_{1}=\left(N_{\alpha_{0}} / H_{1}\right) \cap\left(N_{\beta} / H_{1}\right)$. Hence the index of $P_{\beta} / H_{1}$ in $G_{2} / H_{1}$ is less than or equal to the product of the indices of $N_{\alpha_{0}} / H_{1}$ and $N_{\beta} / H_{1}$ in $G_{2} / H_{1}$ and this product is finite. Since $P_{\beta} / H_{1} \subset N_{\beta} / H_{1} \subset G_{2} / H_{1}$ for every $\beta$, since there are more than $A$ of the $N_{\beta}$, and since there are only a finite number of $N_{\beta} / H_{1}$ between a given $P_{\beta} / H_{1}$ and $G_{2} / H_{1}$, there are more than $A$ of the $P_{\beta}$. Now since there are more than $A$ of the $P_{\beta} / H_{1}$ such that $P_{\beta} / H_{1} \subset N_{\alpha_{0}} / H_{1}$, it follows that $N_{\alpha_{0}} / H_{1}$ has more than $A$ subgroups. But, by (2.1), $\left(N_{\alpha_{0}} / H_{1}\right) \cong\left(G_{2} / H_{1}\right) /\left(H / H_{1}\right) \cong G_{2} / H$, which has at most $A$ subgroups. This contradiction establishes the lemma.

In the following lemma the countable torsion Abelian groups which have $\boldsymbol{\aleph}_{0}$ subgroups are characterized.

Leмma 3. H. $G$ is a countable torsion Abelian group.

C. (i) $G$ has $\aleph_{0}$ subgroups if $G=Z\left(p_{1}^{\infty}\right) \oplus Z\left(p_{2}^{\infty}\right) \oplus \cdots \oplus Z\left(p_{n}^{\infty}\right) \oplus F$, where $p_{i} \neq p_{j}$ for $i \neq j$ and $F$ is finite. ${ }^{3}$ (ii) $G$ has $\aleph$ subgroups otherwise.

${ }^{3}$ Hereafter the form of $G$ given in C.(i) will be called countable form. 
Proof. $G=D \oplus R$, with $D$ divisible and $R$ reduced. Consider the two cases:

CASE 1. $R$ is countable. Write $R$ as a direct sum of primary groups. If there are $\boldsymbol{\aleph}_{0}$ summands of $R$ then $G$ has $\boldsymbol{\aleph}$ subgroups since the direct sum of any collection of the summands forms a subgroup. If $R=R_{p_{1}} \oplus \cdots \oplus R_{p_{n}}$, where the $R_{p_{i}}$ are primary with respect to the prime $p_{i}$, then at least one summand, say $R_{p}$, is countable since $R$ is countable. Now $R_{p}=C_{1} \oplus R_{p}^{\prime}$, where $C_{1}$ is a finite cyclic group, $R_{p}^{\prime}=C_{2} \oplus R_{p}^{\prime \prime}$, and continuing in this way a sequence $\left\{C_{n}\right\}$ of finite cyclic groups is obtained such that no $C_{i}$ is contained in the direct sum of any of the others. Hence the direct sum of any subcollection of these cyclic groups forms a subgroup of $G$ and $G$ has $N$ subgroups.

CASE 2. $R$ is finite. Since $D=Z\left(p_{1}^{\infty}\right) \oplus \cdots \oplus Z\left(p_{n}^{\infty}\right) \oplus \cdots$, it follows from Lemma 1 that if $p_{i}=p_{j}$ for $i \neq j$ then $G$ has $\aleph$ subgroups. If there are $\boldsymbol{N}_{0}$ summands of $D$ then by the reason used twice in Case 1 , $G$ has $\aleph$ subgroups. Otherwise $D=Z\left(p_{1}^{\infty}\right) \oplus \cdots \oplus Z\left(p_{n}^{\infty}\right)$ and $G$ has countable form. This proves (ii).

Now assume that $G$ has countable form. Then $G=Z\left(p_{1}^{\infty}\right) \oplus F_{p_{1}}$ $\oplus \cdots \oplus Z\left(p_{n}^{\infty}\right) \oplus F_{p_{n}} \oplus F_{p_{n+1}} \oplus \cdots \oplus F_{p_{m}}$, where $F_{p_{i}}$ is the primary subgroup of $F$ with respect to the prime $p_{i}$. If $H$ is any subgroup of $G$, then $H=H_{p_{1}} \oplus \cdots \oplus H_{p_{m}}$ and $H_{p_{i}} \subset Z\left(p_{i}^{\infty}\right) \oplus F_{p_{i}}$ for $i=1, \cdots, n$ and $H_{p_{i}} \subset F_{p_{i}}$ for $i=n+1, \cdots, m$. If $K$ is any subgroup of $Z\left(p^{\infty}\right) \oplus F_{p}$ then $K /\left(K \cap Z\left(p^{\infty}\right)\right) \cong\left(K \cup Z\left(p^{\infty}\right)\right) / Z\left(p^{\infty}\right) \subset\left(Z\left(p^{\infty}\right) \oplus F_{p}\right) / Z\left(p^{\infty}\right) \cong F_{p}$. Hence the index of $K \cap Z\left(p^{\infty}\right)$ in $K$ is less than or equal to the order of $F_{p}$, which is finite. Thus $K$ admits the coset decomposition $K=\left(K \cap Z\left(p^{\infty}\right)\right) g_{1}+\cdots+\left(K \cap Z\left(p^{\infty}\right)\right) g_{r}$. Since $Z\left(p^{\infty}\right)$ is countable and has $\aleph_{0}$ subgroups, $K \cap Z\left(p^{\infty}\right)$ and $g_{1}, \cdots, g_{r}$ can be obtained in at most $\boldsymbol{\aleph}_{0}$ ways. Hence $Z\left(p^{\infty}\right) \oplus F_{p}$ has $\boldsymbol{\aleph}_{0}$ subgroups, and, since the number of subgroups of $G$ is equal to the product of the numbers of the subgroups of $Z\left(p_{i}^{\infty}\right) \oplus F_{p_{i}}$ for $i=1, \cdots, n$ and the numbers of the subgroups of $F_{p_{i}}$ for $i=n+1, \cdots, m$, it follows that $G$ has $\aleph_{0}$ subgroups. This completes the proof.

Definition 1. A subgroup $H$ of an Abelian group $G$ is said to be inextensible if $g$ is in $H$ whenever $n g$ is in $H, n$ an integer.

The intersection of a set of inextensible subgroups is inextensible.

Definition 2. The intersection of all the inextensible subgroups containing a given set $S$ of elements of an Abelian group $G$ is said to be the extension $R(S)$ of that set of elements.

Notice that the extension of a set $S$ of elements is precisely the set of all $g$ such that for some integer $n, n g$ is a linear combination of the elements of $S$.

Theorem 1. H. G is a countable Abelian group. $M$ is a maximal set 
of linearly independent elements of $G . B$ is the free group generated by the elements of $M$.

C. (i) $G$ has $\aleph_{0}$ subgroups if the order $o(M)$ of $M$ is finite and $G / B$ has countable form. (ii) $G$ has $\aleph$ subgroups otherwise.

Proof. If $o(M)=\boldsymbol{\aleph}_{0}$, then $G$ has $\boldsymbol{N}$ subgroups since any two distinct subsets of $M$ generate distinct subgroups of $G$. For every $g$ in $G$ there is an integer $n$ such that $n g$ is in $B$; hence $G / B$ is torsion and by Lemma $3, G$ has $N$ subgroups if $G / B$ does not have countable form. This proves (ii).

Conversely, assume that $o(M)<\boldsymbol{N}_{0}$ and $G / B$ has countable form. Let $H$ be any subgroup of $G$ such that $H \nsubseteq B$ and $B \nsubseteq H$. Consider $R(H \cap B)$. By the remark following Definition 2, $R(H \cap B) /(H \cap B)$ is torsion. Also, since for every $g$ in $G$ there is some integer $m$ such that $m g$ is in $B, R(H \cap B) /(R(H \cap B) \cap B)$ is torsion. However $R(H \cap B) /(R(H \cap B) \cap B) \cong(R(H \cap B) \cup B) / B \subset G / B$; hence $R(H \cap B) /(R(H \cap B) \cap B)$ has at most $\boldsymbol{\aleph}_{0}$ subgroups. Both $R(H \cap B)$ $\cap B$ and $H \cap B$ are free groups. Also, since for each generator $g_{i}$ of $R(H \cap B) \cap B$ there is an integer $n_{i}$ such that $n_{i} g_{i}$ is in $H \cap B$, $(R(H \cap B) \cap B) /(H \cap B)$ is finite. Further

$$
\begin{aligned}
(R(H \cap B) /(H \cap B)) /((R(H \cap B) & \cap B) /(H \cap B)) \\
& \cong R(H \cap B) /(R(H \cap B) \cap B),
\end{aligned}
$$

which has at most $\boldsymbol{\aleph}_{0}$ subgroups. Hence by Lemma $2, R(H \cap B) /(H \cap B)$ has at most $\aleph_{0}$ subgroups. For any $h$ in $H, n h$ is in $B$ for some integer $n$; hence $n h$ is in $H \cap B$ and this implies, by the remark following Definition 2, that $h$ is in $R(H \cap B)$. Thus $H \cap B \subset H \subset R(H \cap B)$. Thus it has been proved that for each subgroup $B^{\prime}$ of $B$ there are at most $\aleph_{0}$ subgroups $H_{\alpha}$ of $G$ such that $H_{\alpha} \cap B=B^{\prime}$. Since $B$ has $\aleph_{0}$ subgroups it follows that $G$ has at most $\aleph_{0}$ subgroups. Also, since $G$ is countable, $G$ has at least $\boldsymbol{\aleph}_{0}$ subgroups. Therefore $G$ has $\aleph_{0}$ subgroups, which was to be proved.

Corollary 1. H. Same as in Theorem 1.

C. (i) If $o(M)=\boldsymbol{\aleph}_{0}$ or $G / B$ is not of countable form then $G$ has $\aleph$ infinite subgroups. (ii) If $o(M)<\aleph_{0}$ and $G / B$ has countable form, then $G$ has $\aleph_{0}$ infinite subgroups unless $G=Z\left(p^{\infty}\right) \oplus F$, where $F$ is finite. (iii) If $G=Z\left(p^{\infty}\right) \oplus F$, with $F$ finite, then the number of infinite subgroups of $G$ is the same as the number of subgroups of $F$.

Proof. (i) and (ii) are immediate from the proof of Lemma 3 and from Theorem 1. For (iii), notice that if $H$ is an infinite subgroup of $G$, then $H \cap Z\left(p^{\infty}\right)=Z\left(p^{\infty}\right)$, for otherwise $H \cap Z\left(p^{\infty}\right)$ is finite and hence 
$H$ is finite. Hence the number of infinite subgroups of $Z\left(p^{\infty}\right) \oplus F$ is the same as the number of subgroups of $\left(Z\left(p^{\infty}\right) \oplus F\right) / Z\left(p^{\infty}\right) \cong F$.

Corollary 2. H. G is a countable Abelian group. $T$ is the torsion subgroup of $G$. $G$ has less than $\aleph$ subgroups.

C. $T$ is a direct summand of $G$.

Proof. Since $G$ has less than $\aleph$ subgroups, $T$ has countable form, $T=Z\left(p_{1}^{\infty}\right) \oplus \cdots \oplus Z\left(p_{n}^{\infty}\right) \oplus F$. Now $F$ is a finite pure subgroup of $G$, hence $G=F \oplus G^{\prime}$. Also, since $Z\left(p_{i}^{\infty}\right) \cap F=0, G^{\prime}=Z\left(p_{1}^{\infty}\right) \oplus \cdots \oplus Z\left(p_{n}^{\infty}\right)$ $\oplus G^{\prime \prime}$, i.e. $G=T \oplus G^{\prime \prime}$.

One may be tempted to conjecture that if $o(M)<\boldsymbol{\aleph}_{0}$ and $G$ has $\boldsymbol{\aleph}_{0}$ subgroups, where $G$ is a countable Abelian group, then $G$ is a direct sum of rational groups (subgroups of the additive group of rationals, $R^{+}$, or subgroups of $R^{+} / Z$, where $Z$ is the additive group of integers). This conjecture would be defeated by the following example which is a modification of the example given in the proof of $[1$, Theorem 19].

Let $u$ and $v$ be two symbols, let $p$ be a prime, and let $G$ be the group of all finite linear combinations over the integers of the expressions $v$, $w_{1} / p, w_{2} / p^{3}, \cdots, w_{n} / p^{((n-1) / 2)(n+2)+1}, \cdots$ where $w_{n}=u+\left(1+p^{2}\right.$ $\left.+p^{5}+\cdots+p^{((n-1) / 2)(n+2)}\right) v$. In this example it is possible to take $M=\{u, v\}$ and it is a straightforward matter to show that $G / B$ $\cong Z\left(p^{\infty}\right)$. Also, assuming that $G$ is the direct sum of rational groups it is easy to show, by a calculation similar to that in $[1]$, that $G / B$ is finite, a contradiction.

The following theorem concerns modules over a principal ideal ring. Notice that an Abelian group of order $A>\boldsymbol{N}_{0}$ is a module over the integers and there are only $\boldsymbol{\aleph}_{0}$ integers. Hence the theorem of Scott which was mentioned in the Introduction is a corollary of the following theorem.

TheOREM 2. H. $M$ is a module over a principal ideal ring $S . o(S)$ $<o(M) . R$ is the set of submodules of $M$ which are of order $o(M) . D$ is the intersection of all the submodules in $R$.

C. (i) $o(R)=2^{o(M)}$. (ii) $D$ is the identity.

Proof. If $o(M)>\boldsymbol{\aleph}_{0}$, the proof of this theorem is obtained by translating the proof of Scott's theorem into module language and for this reason it will be omitted. If $o(M)=\boldsymbol{\aleph}_{0}$, then $o(S)$ is finite and $S$ is a field. Hence $M$ is the direct sum of $\aleph_{0}$ copies of $S$ and the theorem follows.

3. The order of the automorphism group. In this article it will be 
shown that the order of the automorphism group of a countable torsion Abelian group is $\aleph$. The proof will depend on the proof of Ulm's theorem as given in [1, Theorem 14].

Theorem 3. H. $G$ is a countable torsion Abelian group. $A(G)$ is the automorphism group of $G$.

C. $o(A(G))=\aleph$.

Proof. Clearly, $o(A(G)) \leqq \aleph . G=D \oplus R$. If $D \neq 0$ then $D$ is the direct sum of $Z\left(p^{\infty}\right)$ groups. Also, an automorphism of $Z\left(p^{\infty}\right)$ is given by a sequence of correspondences $1 / p \rightarrow h / p, 0 \neq h<p ; 1 / p^{2} \rightarrow k / p^{2}$, $k<p^{2}, k \equiv h(\bmod p) ; \cdots$ and there are $\aleph$ such sequences. Since the automorphism group of a weak direct sum of Abelian groups contains a subgroup which is isomorphic to the strong direct sum of the automorphism groups of the summands (hereafter this will be called property S), it follows that $o(A(G))=\aleph$ in case $D \neq 0$. If $D=0$ then $G=R$ and $R$ is a direct sum of primary groups. If there are $\aleph_{0}$ summands of $R$, then each summand has a finite cyclic direct summand; and all but at most one of them will be of order greater than two. Hence the automorphism group of each finite cyclic direct summand will be of order at least two. In this case the theorem follows from property $\mathrm{S}$. The only case remaining to be considered is the one in which $R$ is the direct sum of a finite number of primary groups. In this case there will be a prime $p$ such that the order of the corresponding primary group is $\aleph_{0}$. Hence it must be proved that if $G$ is a countable reduced primary Abelian group, then $o(A(G))=\aleph$. This follows from the proof of Ulm's theorem in [1], for in building up an automorphism it is possible to make the extension described in [1] in two ways at all but at most one stage. In the terminology of [1], the element $w$ can be changed by any element of $P_{\lambda-1}$ provided the height of $x$ is at most $\lambda-2$. If the height of $x$ is $\lambda-1$ then $w$, an element of $P_{\lambda-1}$, may be changed by any element of $P_{\lambda-1}$. This is impossible only when $P_{\lambda-1}$ is cyclic of order 2 . Hence $w$ may be chosen in two ways at all but at most one step, and an automorphism can be built up in $\boldsymbol{N}$ ways. This proves the theorem.

\section{BIBLIOGRAPHY}

1. I. Kaplansky, Infinite Abelian groups, Ann Arbor, University of Michigan Press, 1954.

2. W. R. Scott, Groups and cardinal numbers, Amer. J. Math. vol. 74 (1952) pp. 187-197.

UNIVERSITY OF KANSAS 